\title{
THE PAIR CORRELATION OF ZEROS OF DIRICHLET $L$ FUNCTIONS AND PRIMES IN ARITHMETIC PROGRESSIONS
}

\section{YALÇIN YILDIRIM}

We define a function which correlates the zeros of two Dirichlet $L$-functions to the modulus $q$ and we prove an asymptotic estimate for averages of the pair correlation functions over all pairs of characters to (mod $q$ ). An analogue of Montgomery's pair correlation conjecture is formulated as to how this estimate can be extended to a greater domain for the parameters that are involved. Based on this conjecture we obtain results about the distribution of primes in an arithmetic progression (to a prime modulus $q$ ) and gaps between such primes.

\section{Introduction and statement of results}

In 1973 Montgomery's approach [7] provided a new direction for research on the Riemann zeta-function, $\zeta(s)$, and the distribution of primes. Assuming the Riemann Hypothesis Montgomery defined the pair correlation function of the critical zeros of $\zeta(s)$

$$
F(\alpha, T)=\left(\frac{T \log T}{2 \pi}\right)^{-1} \sum_{\substack{\alpha<\gamma, \gamma^{\prime} \leq T \\\left(\frac{1}{2}+i \gamma\right)=0 \\\left\langle\left(\frac{1}{2}+i \gamma^{\prime}\right)=0\right.}} T^{i \alpha\left(\gamma-\gamma^{\prime}\right)} w\left(\gamma-\gamma^{\prime}\right)
$$

(where $w(u)=\frac{4}{4+u^{2}}$ is a weighting function which serves to diminish the contribution from those pairs of zeros with large differences) and he proved that (see [7] and [2])

$$
F(\alpha, T)=(1+o(1)) T^{-2 \alpha} \log T+\alpha+o(1)
$$

as $T \rightarrow \infty$, uniformly for $0 \leq \alpha \leq 1$. Montgomery also conjectured that

$$
F(\alpha, T)=1+o(1)
$$

for $\alpha \geq 1$, uniformly in bounded intervals. This statement has become known as the pair correlation conjecture. He then used (3) to show that almost all zeros of $\zeta(s)$ are simple.

Assuming $\mathrm{RH}$ and the pair correlation conjecture in various forms Heath-Brown [5] proved that

$$
\sum_{\substack{p_{n} \leq x \\ d_{n} \geq \Delta}} d_{n} \ll \frac{x}{\Delta} \log x
$$




\section{YALÇIN YILDIRIM}

(where $d_{n}=p_{n+1}-p_{n}$ and $p_{n}$ is the n-th prime) and that for functions $f$ such that $f(x) \rightarrow \infty$ as $x \rightarrow \infty$, almost all intervals $[x, x+f(x) \log x]$ contain a prime. HeathBrown also showed that

$$
\liminf _{n \rightarrow \infty} \frac{p_{n+1}-p_{n}}{\log p_{n}}=0 .
$$

Goldston and Heath-Brown [3] proved that RH and the pair correlation conjecture together imply $d_{n}=o\left(\left(p_{n} \log p_{n}\right)^{\frac{1}{2}}\right)$.

Moreover, Goldston and Montgomery established an equivalence between an asymptotic result for the distribution of primes and the pair correlation conjecture (see [4], Theorem 2).

In this paper we apply the ideas of the pair correlation conjecture to the distribution of primes in an arithmetic progression. Let,

$$
G_{\chi_{1}, \chi_{2}}(x, T)=2 \pi \sum_{0<\gamma_{1}, \gamma_{2} \leq T} x^{i\left(\gamma_{1}-\gamma_{2}\right)} w\left(\gamma_{1}-\gamma_{2}\right)
$$

where, assuming GRH, $\frac{1}{2}+i \gamma_{j}$ runs through the zeros of the Dirichlet $L$-functions $L\left(s, \chi_{j}\right)$, $(j=1,2)$, and $\chi_{j}$ are characters to the modulus $q$. One may say that $G_{\chi_{1}, \chi_{2}}(x, T)$ correlates the critical zeros of $L\left(s, \chi_{1}\right)$ with those of $L\left(s, \chi_{2}\right)$.

In the following we suppose $(a, q)=1$. Capital letters $A, B, C$ will denote arbitrary fixed positive numbers. We prove

Theorem 1. Assume GRH. As $x \rightarrow \infty$ we have, uniformly for

$$
1 \leq q \leq x^{\frac{1}{2}} \log ^{-3} x
$$

when $T$ is in the range

$$
\begin{gathered}
\frac{x}{q} \log x \leq T \leq e^{\sqrt[4]{x}} \\
\sum_{\chi_{1}, \chi_{2}(\bmod q)} \bar{\chi}_{1}(a) \chi_{2}(a) G_{\chi_{1}, \chi_{2}}(x, T) \sim \phi(q) T \log x .
\end{gathered}
$$

For $T$ smaller relative to $x$ we assume the following

Conjecture. Under GRH, as $x \rightarrow \infty$, it holds uniformly for

$$
q \leq \min \left(x^{\frac{1}{2}} \log ^{-3} x, x^{1-\eta} \log x\right)
$$

and

$$
x^{\eta} \leq T \leq \frac{x}{q} \log x
$$

where $q$ is prime or 1 and $0<\eta \leq 1$ is fixed, that

$$
\sum_{\chi_{1}, \chi_{2}(\bmod q)} \bar{\chi}_{1}(a) \chi_{2}(a) G_{\chi_{1}, \chi_{2}}(x, T) \sim \phi(q) T \log q T
$$




\section{YALÇIN YILDIRIM}

The restriction to prime moduli $q$ in the Conjecture is made to avoid the presence of imprimitive characters.

Let

$$
\psi(x ; q, a)=\sum_{\substack{n \leq x \\ n \equiv a(\bmod q)}} \Lambda(n)
$$

where $\Lambda$ is the von Mangoldt function. Upon the Generalized Riemann Hypothesis (GRH) the prime number theorem for arithmetic progressions is, for $q \leq x,([1], p .125)$

$$
\psi(x ; q, a)=\frac{x}{\phi(q)}+O\left(x^{\frac{1}{2}} \log ^{2} x\right)
$$

Assuming the Conjecture we obtain the following asymptotic result for an individual arithmetic progression with prime modulus.

Theorem 2. Assume GRH. Let $\alpha_{1}$ and $\alpha_{2}$ be fixed, $0<\alpha_{1} \leq \alpha \leq \alpha_{2} \leq 1$ and $\delta=x^{-\alpha}$. Also let $0<\eta<\alpha_{1}$ be fixed. Assume that the Conjecture holds uniformly for

$$
q \leq \min \left(x^{\frac{1}{2}} \delta^{\frac{1}{2}}(\log x)^{A}, \delta^{-1} x^{-\eta}\right)
$$

where $q$ is prime or 1 and

$$
\frac{x^{\alpha_{1}}}{\phi(q)} \log ^{-3} x \leq T \leq \phi(q) x^{\alpha_{2}} \log ^{3} x
$$

Then

$$
\int_{x}^{2 x}\left\{\psi(u+u \delta ; q, a)-\psi(u ; q, a)-\frac{u \delta}{\phi(q)}\right\}^{2} d u \sim \frac{3}{2} \frac{\delta x^{2}}{\phi(q)} \log \frac{q}{\delta},
$$

as $x \rightarrow \infty$, uniformly for $x^{-\alpha_{2}} \leq \delta \leq x^{-\alpha_{1}}$ and such moduli $q$.

The following Corollaries, all resting on GRH, may be deduced from Theorem 2 . Let $p_{n}(a, q)$ denote the $n$-th prime congruent to $a(\bmod q)$ and $d_{n}(a, q)=p_{n+1}(a, q)-p_{n}(a, q)$. The modulus $q$ is always supposed to be prime or 1 . For $q=1$ all of the results presented here yield the known estimates $([2],[3],\{4],\{5],[7])$ mentioned above. By the weaker form of the Conjecture it is meant that there is $\ll$ in place of the asymptotic estimate in the Conjecture. This implies the same change in the estimate for the integral in Theorem 2.

Corollary 1. Let $4 \leq \Delta \leq x^{1-\varepsilon}$ and $\varepsilon>0$ be fixed as $x \rightarrow \infty$. Also let $0<\eta<$ $1-\frac{\log \Delta}{\log x}$ be fixed. Assume the weaker form of the Conjecture for

$$
q \leq \min \left(\Delta^{\frac{1}{2}}(\log x)^{A}, \frac{x^{1-\eta}}{\Delta} \log ^{-3} x\right)
$$

and

$$
\frac{x}{\phi(q) \Delta} \log ^{-3} x \leq T \leq \frac{\phi(q) x}{\Delta} \log ^{3} x
$$




\section{YALÇIN YILDIRIM}

Then

$$
\sum_{\substack{x \leq p_{n}(a, q) \leq \frac{3}{a} x \\ d_{n}(\alpha, q) \geq \Delta}} d_{n}(a, q) \ll \phi(q) \frac{x}{\Delta} \log x
$$

uniformly for such $q$.

Corollary 1 is non-trivial if $q \log x \leq \Delta$. It follows readily from Corollary 1 that

Corollary 2. Let $q \leq(\log x)^{A}$ and $f(x) \rightarrow \infty$ arbitrarily slowly as $x \rightarrow \infty$. Assume the weaker form of the Conjecture for

$$
\frac{x(\log x)^{-(4+2 A)}}{f(x)} \leq T \leq x \log ^{2} x
$$

Then

$$
\sum_{\substack{x \leq p_{n}(a, q) \leq \sum_{0}^{3} x \\ d_{n}(a, q) \geq \phi(q) f(x) \log x}} d_{n}(a, q) \ll \frac{x}{f(x)} .
$$

Hence for $q \leq(\log x)^{A}$ almost all intervals $[x, x+\phi(q) f(x) \log x]$ contain a prime congruent to $a(\bmod q)$.

Corollary 3. Assume that the asymptotic estimate of the Conjecture holds uniformly for $q \leq x^{\frac{1}{3}-\eta}$ and

$$
\sqrt{\frac{x}{q^{3}}} \log ^{-\frac{\pi}{2}} x \leq T \leq \sqrt{q x} \log ^{2} x
$$

Then, for $p_{n}(a, q) \asymp x$,

$$
d_{n}(a, q)=o\left(\phi(q) p_{n}(a, q) \log p_{n}(a, q)\right)^{\frac{1}{2}}
$$

uniformly for such $q$.

Corollary 4. Assume that the asymptotic estimate of the Conjecture holds uniformly for $q \leq(\log x)^{A}$ and

$$
x(\log x)^{-(4+2 A)} \leq T \leq x \log ^{3} x,
$$

as $x \rightarrow \infty$. Let $p_{n}(a, q) \asymp x$. Then

$$
\liminf _{n \rightarrow \infty} \frac{d_{n}(a, q)}{\phi(q) \log p_{n}(a, q)}=0
$$

\section{Explicit formulae and the proof of Theorem 1}

Paralleling Landau's derivation of an explicit formula ([6], p. 353) that provides a link between the zeros of $\zeta(s)$ and primes one gets in the case of Dirichlet $L$-functions

$$
\sum_{n \leq x}^{\prime} \frac{\Lambda(n) \chi(n)}{n^{r}}=\sum_{\ell=0}^{\infty} \frac{x^{-2 \ell-r-a}}{2 \ell+r+a}-\sum_{\rho} \frac{x^{\rho-r}}{\rho-r}-\frac{L^{\prime}}{L}(s, \chi),
$$




\section{YALÇIN YILDIRIM}

for $x>1$ (the prime on the summation means only half of the term with $n=x$ is included in the sum) and $r \neq \rho, r \neq-(2 q+a)$ where for primitive $\chi(\bmod q)$

$$
\mathbf{a}= \begin{cases}0, & \text { if } \quad \chi(-1)=1 \\ 1, & \text { if } \quad \chi(-1)=-1\end{cases}
$$

The sum over the non-trivial zeros $\rho$ of $L(s, \chi)$ in $(6)$ is interpreted in the symmetric sense as $\lim _{T \rightarrow \infty} \sum|\gamma|<T \frac{x^{\rho-r}}{p-r}$.

The explicit formula (6) combined with the functional equation of $\frac{L^{\prime}}{L}(s, \chi)$, as in the proof of the Lemma of Montgomery [7], leads to another explicit formula

$$
\begin{gathered}
(2 \sigma-1) \sum_{p} \frac{x^{i \gamma}}{\left(\sigma-\frac{1}{2}\right)^{2}+(t-\gamma)^{2}}=-x^{-\frac{1}{2}}\left[\sum_{n \leq x} \Lambda(n) \chi(n)\left(\frac{x}{n}\right)^{1-\sigma+i t}\right. \\
\left.+\sum_{n>x} \Lambda(n) \chi(n)\left(\frac{x}{n}\right)^{\sigma+i t}\right] \\
+x^{\frac{1}{2}-\sigma+i t}\left(\log q \tau+O_{\sigma}(1)\right) \\
+O\left(x^{-\frac{1}{2}-a} \tau^{-1}\right) .
\end{gathered}
$$

This last explicit formula is valid, under GRH for $L(s, \chi)$, for all $x \geq 1$.

From (7) we can write for $(a, q)=1$

$$
\begin{aligned}
& \left|\sum_{\chi(\bmod q)} \bar{\chi}(a) \sum_{\gamma} \frac{2 x^{i \gamma}}{1+(t-\gamma)^{2}}\right|^{2}= \\
& \mid \sum_{\chi(\bmod q)} \bar{\chi}(a)\left\{-x^{-\frac{1}{2}}\left(\sum_{n \leq x} \Lambda(n) \chi(n)\left(\frac{x}{n}\right)^{-\frac{1}{2}+i t}+\sum_{n>x} \Lambda(n) \chi(n)\left(\frac{x}{n}\right)^{\frac{3}{2}+i t}\right)\right. \\
+ & x^{-1+i t}\left(\log q^{\prime} \tau+O(1)\right)+O\left(x^{-\frac{1}{2}-\mathbf{a}} \tau^{-1}\right) \\
+ & \left.x^{\frac{1}{2}}\left(\sum_{\substack{n \leq x \\
x(n) \neq \chi^{\prime}(n)}} \Lambda(n) \chi^{\prime}(n)\left(\frac{x}{n}\right)^{-\frac{1}{2}+i t}+\sum_{\substack{n>x \\
\chi(n) \neq \chi^{\prime}(n)}} \Lambda(n) \chi^{\prime}(n)\left(\frac{x}{n}\right)^{\frac{3}{2}+i t}\right)\right\}\left.\right|^{2},
\end{aligned}
$$

where $\chi(\bmod q)$ is a character induced by the primitive character $\chi^{\prime}\left(\bmod q^{\prime}\right)$. The last term in the parantheses is a correction term for nonprimitive $\chi$. The terms with $\chi(n) \neq$ $\chi^{\prime}(n)$ can exist only when $(n, q)>1$ and when summed over all $\chi(\bmod q)$ the contribution of the correction term vanishes.

We integrate both sides of (8) from $t=0$ to $t=T$. From the left side we obtain (cf. Eq.s (23)-(26) [7] and (4) above)

$$
\begin{aligned}
& \int_{0}^{T} \sum_{\chi_{1}, \chi_{2}} \bar{\chi}_{1}(a) \chi_{2}(a) \sum_{\gamma_{1}, \gamma_{2}} \frac{4 x^{i\left(\gamma_{1}-\gamma_{2}\right)}}{\left[1+\left(t-\gamma_{1}\right)^{2}\right]\left[1+\left(t-\gamma_{2}\right)^{2}\right]} d t= \\
& \sum_{\chi_{1}, \chi_{2}} \bar{\chi}_{1}(a) \chi_{2}(a) G_{\chi_{1}, \chi_{2}}(x, T)+O\left(\phi(q)^{2} \log T \log ^{2} q T\right) .
\end{aligned}
$$


Writing

$$
a_{n}=\left\{\begin{array}{lll}
\Lambda(n)\left(\frac{x}{n}\right)^{-\frac{1}{2}}, & \text { if } & n \leq x, \\
\Lambda(n)\left(\frac{x}{n}\right)^{\frac{3}{2}}, & \text { if } & n>x,
\end{array}\right.
$$

the first term in parentheses on the right-hand side of (8) contibutes

$$
\frac{\phi(q)^{2}}{x}\left|\sum_{n \equiv a(\bmod q)} a_{n} n^{-i t}\right|^{2}
$$

By the Montgomery-Vaughan mean value theorem for Dirichlet series ([8]) in the form

$$
\left.\int_{0}^{T} \sum_{n \equiv a(\bmod q)} a_{n} n^{-i t}\right|^{2} d t=T \sum_{n \equiv a(\bmod q)}\left|a_{n}\right|^{2}+O\left(q \sum_{\substack{n<q \\ n \equiv a(\bmod q)}} \frac{\left|a_{n}\right|^{2}}{n}+\frac{1}{q} \sum_{\substack{n>q \\ n \equiv a(\bmod q)}} n\left|a_{n}\right|^{2}\right)
$$

and using (5) we find that for $q \leq x^{\frac{1}{2}} \log ^{-3} x$ as $T$, and $x \rightarrow \infty$,

$$
\frac{\phi(q)^{2}}{x} \int_{0}^{T}\left|\sum_{n \equiv a(\bmod q)} a_{n} n^{-i t}\right|^{2} d t=\phi(q) T \log x(1+o(1))+O(x \log x) .
$$

The remaining contributions to the integral of the right side of (8) are

$$
\begin{aligned}
& \int_{0}^{T} \frac{\phi(q)^{2}}{x} \log ^{2} q \tau d t \ll \phi(q)^{2} \frac{T}{x} \log ^{2} q T \\
& \int_{0}^{T} \frac{\phi(q)^{2}}{x \tau^{2}} d t \ll \frac{\phi(q)^{2}}{x}
\end{aligned}
$$

and the cross-terms. If $(\log T)^{4} \leq x$ then the main term is $(10)$ and Theorem 1 holds.

\section{Proof of Theorem 2}

The method of Goldston and Montgomery [4] about primes in short intervals based on the pair correlation conjecture for $\zeta(s)$ is adaptable to primes in arithmetic progressions in short intervals. We have the following lemmas the first two of which have been slightly modified from their original statements in [4].

Lemma 1.([4] Lemma 2) Let $\phi$ satisfy $1 \leq \phi \leq\left(\frac{1}{\kappa}\right)^{B}$ where $B \geq 0$ is fixed as $\kappa \rightarrow 0^{+}$. Let $f(t)$ be a continuous, non-negative function defined for $t>0$ such that

$$
f(t) \ll \phi^{2} \log ^{2} \phi \tau .
$$

Assume further that

$$
J(T)=\int_{0}^{T} f(t) d t=(1+\varepsilon(T)) \phi T \log \phi T,
$$




\section{YALÇIN YILDIRIM}

$|\varepsilon(T)|$ being small (that is, given $\varepsilon_{0}>0,|\varepsilon(T)|<\varepsilon_{0}$ for sufficiently large $T$ ) uniformly for

$$
\frac{1}{\phi \kappa \log ^{2} \kappa} \leq T \leq \frac{\phi}{\kappa} \log ^{2} \frac{\phi}{\kappa} \text {. }
$$

Then,

$$
\int_{0}^{\infty}\left(\frac{\sin \kappa u}{u}\right)^{2} f(u) d u \sim\left(\frac{\pi}{2}+\varepsilon^{\prime}(\kappa)\right) \phi \kappa \log \frac{\phi}{\kappa},
$$

where $\left|\varepsilon^{\prime}(\kappa)\right|$ is small as $\kappa \rightarrow 0^{+}$(that is, given $\varepsilon_{0}>0,\left|\varepsilon^{\prime}(\kappa)\right|<\varepsilon_{0}$ for sufficiently small $\kappa)$. This result is uniform in $\phi$.

Lemma 2.([4] Lemma 10) Let $\omega(s)=\frac{(1+\delta)^{s}-1}{s}$ where $\delta \in(0,1]$. Then

$$
\begin{aligned}
& \int_{-\infty}^{\infty}|\omega(i t)|^{2}\left|\sum_{\chi(\bmod q)} \bar{\chi}(a) \sum_{\gamma} \frac{x^{i \gamma}}{1+(t-\gamma)^{2}}\right|^{2} d t= \\
& \int_{-\infty}^{\infty}\left|\sum_{x(\bmod q)} \bar{\chi}(a) \sum_{|\gamma| \leq Z} \frac{\omega\left(\frac{1}{2}+i \gamma\right) x^{i \gamma}}{1+(t-\gamma)^{2}}\right|^{2} d t \\
& +O\left(\phi(q)^{2} \delta^{2} \log ^{3} \frac{2 q}{\delta}\right)+O\left(\phi(q)^{2} Z^{-1} \log ^{3} Z\right)
\end{aligned}
$$

provided that $Z \geq \delta^{-1}$.

Lemma 3.([4] Lemma 1) If $\int_{-\infty}^{\infty} e^{-2|y|} f(Y+y) d y=1+\epsilon(Y)$ and if $f(y) \geq 0$ for all $y$, then for any Riemann integrable function $R(y)$

$$
\int_{a}^{b} R(y) f(Y+y) d y=\left(\int_{a}^{b} R(y) d y\right)\left(1+\epsilon^{\prime}(Y)\right) .
$$

If $R$ is fixed then, as $Y \rightarrow \infty,\left|\epsilon^{\prime}(Y)\right|$ is small if $|\epsilon(y)|$ is amall uniformly for $Y+a-1 \leq$ $y \leq Y+b+1$.

In Lemma 1 we take

$$
f(t)=\left|\sum_{\chi(\bmod q)} \bar{\chi}(a) \sum_{\gamma} \frac{2 x^{i \gamma}}{1+(t-\gamma)^{2}}\right|^{2}
$$

(trivially $\left.f(t) \ll \phi(q)^{2} \log ^{2} q \tau\right)$ and $\phi=\phi(q)$. Let $\kappa=\frac{1}{2} \log (1+\delta)$ and assume that the asymptotic value $\phi(q) T \log q T$ of the Conjecture is valid, uniformly for

$$
\frac{1}{\phi(q) \kappa \log ^{2} \kappa} \leq T \leq \frac{\phi(q)}{\kappa} \log ^{2} \frac{q}{\kappa}
$$

as $\kappa \rightarrow 0^{+}$. This range of $T$ is consistent with the range of $T$ for which we may assume the asymptotic estimate of the Conjecture and Theorem 1, if for $0<\eta<\alpha_{1}$

$$
q \leq \min \left(x^{\frac{1}{2}} \kappa^{\frac{1}{2}}(\log x)^{A}, \kappa^{-1} x^{-\eta}\right) .
$$


For such $T$ and $q$ we have, as $x \rightarrow \infty$,

$$
\int_{0}^{T} f(t) d t \sim \phi(q) T \log q T
$$

and therefore by Lemma 1 as $\kappa \rightarrow 0^{+}$

$$
\int_{0}^{\infty}\left(\frac{\sin \kappa t}{t}\right)^{2} f(t) d t \sim \frac{\pi}{2} \phi(q) \kappa \log \frac{q}{\kappa} .
$$

But $\left(\frac{\sin \kappa t}{t}\right)=\frac{\omega(i t)}{4}$ and applying Lemma 2 with $Z=x(\log x)^{C}, C>3$ and fixed,

$$
\int_{-\infty}^{\infty}\left|\sum_{\chi(\bmod q)} \bar{\chi}(a) \sum_{|\gamma| \leq Z} \frac{\omega(\rho) x^{i \gamma}}{1+(t-\gamma)^{2}}\right|^{2} d t \sim \frac{\pi}{2} \phi(q) \delta \log \frac{q}{\delta} .
$$

By Plancherel's identity the last integral is transformed into

$$
\pi^{2} \int_{-\infty}^{\infty}\left|\sum_{\chi(\bmod q)} \bar{\chi}(a) \sum_{|\gamma| \leq Z} \omega(\rho) x^{i \gamma} e(-\gamma u)^{2}\right|^{2} e^{-4 \pi|u|} d u .
$$

Let $Y=\log x$ and $y=-2 \pi u$, so that $x \rightarrow \infty$

$$
\int_{-\infty}^{\infty}\left|\sum_{\chi(\bmod q)} \bar{\chi}(a) \sum_{|\gamma| \leq Z} \omega(\rho) e^{i \gamma(Y+z)}\right|^{2} e^{-2|y|} d y \sim \phi(q) \delta \log \frac{q}{\delta} .
$$

We now use Lemma 3 with

$$
R(y)= \begin{cases}e^{2 y}, & \text { if } 0 \leq y \leq \log 2 \\ 0, & \text { otherwise }\end{cases}
$$

and letting $u=e^{Y+y}$ we have

$$
\int_{x}^{2 x}\left|\sum_{x(\bmod q)} \bar{\chi}(a) \sum_{|\gamma| \leq Z} \omega(\rho) u^{\rho}\right|^{2} d u \sim \frac{3}{2} \phi(q) x^{2} \delta \log \frac{q}{\delta} .
$$

Let now

$$
S_{x}= \begin{cases}1, & \text { if } x=\chi_{0}, \\ 0, & \text { otherwise }\end{cases}
$$

We recall the identity

$$
\begin{aligned}
& \int\left\{\psi((1+\delta) u ; q, a)-\psi(u ; q, a)-\frac{u \delta}{\phi(q)}\right\}^{2} d u= \\
& \frac{1}{\phi(q)^{2}} \sum_{\chi_{1}, \chi_{2}(\bmod q)} \bar{\chi}_{1}(a) \chi_{2}(a) \int\left\{\psi\left((1+\delta) u ; \chi_{1}\right)-\psi\left(u ; \chi_{1}\right)-\delta u S_{\chi_{1}}\right\} \\
& \left.\quad \cdot\left\{\psi(1+\delta) u ; \bar{\chi}_{2}\right)-\psi\left(u ; \bar{\chi}_{2}\right)-\delta u S_{\chi_{2}}\right\} d u
\end{aligned}
$$




\section{YALÇIN YILDIRIM}

and the explicit formula ([D], p. 117)

$$
\begin{aligned}
\psi((1+\delta) u ; \chi)-\psi(u ; \chi)-\delta u S_{\chi}= & -\sum_{|\gamma| \leq Z} \omega(\rho) u^{\rho}+O(\delta)+O\left(\frac{u}{Z} \log ^{2} u q Z\right) \\
& +O\left(\log u \cdot \min \left(1, \frac{u}{Z}\left(\frac{1}{\|u\|}+\frac{1}{\|(1+\delta) u\|}\right)\right)\right) \\
& + \text { correction for nonprimitive } \chi
\end{aligned}
$$

We insert (14) in (13) and when the sums over $\chi_{1}, \chi_{2}$ are carried out the correction terms for nonprimitive characters disappear as in (8). Given $A>0$ we choose $C=C(A)$ (recall that $Z=x(\log x)^{C}$ so that the integral in (12) multiplied by $\frac{1}{\phi(q)^{2}}$ is the main term and all other terms are $o\left(\frac{x^{2} \delta}{\phi(g)} \log x\right)$ as $x \rightarrow \infty$, uniformly for $q$ subject to (11). The proof of Theorem 2 is now complete.

We observe that Theorem 2 remains valid if $\psi$ 's are replaced by $\theta$ 's, i.e. only the contribution from the primes is counted. We will abbreviate $p_{n}(a, q)$ and $d_{n}(a, q)$ as $p_{n}$ and $d_{n}$. To deduce Corollary 1 let $x \leq p_{n} \leq \frac{3}{2} x, \Delta=4 \delta x$ and $\Delta \leq d_{n}(a, q)$. Then $\theta(u+u \delta ; q, a)=\theta(u ; q, a)$ for $p_{n} \leq u \leq p_{n}+\frac{d_{n}}{2}$. By Theorem 2, which holds for $q$ in the specified range,

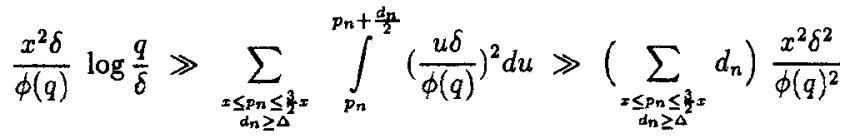

This proves Corollary 1.

To show Corollary 3 suppose that the assertion is false, given any fixed $\epsilon>0$ there exist $p_{n}$ and $p_{n+1}$ such that $x \leq p_{n}<p_{n+1} \leq\left(1+\epsilon^{\frac{3}{2}}\right) x$ and $d_{n} \geq \epsilon \sqrt{\phi(q) x \log x}$ however large $x$ may be. Let $\delta=\frac{\epsilon}{3} \sqrt{\frac{\phi(q) \log x}{x}}$ and $H=3 x \delta$. The range in which the Conjecture is assumed corresponds to this value of $\delta$. Then from Theorem 2

$$
\int_{x}^{\left(1+\varepsilon^{\frac{3}{2}}\right) x}\left\{\theta((u+u \delta) ; q, a)-\theta(u ; q, a)-\frac{u \delta}{\phi(q)}\right\}^{2} d u \ll \epsilon^{\frac{3}{2}} \frac{\delta x^{2}}{\phi(q)} \log \frac{q}{\delta} .
$$

We also have

$$
\int_{p_{n}}^{p_{n+1}-\frac{H}{2}}\left\{\theta((u+u \delta) ; q, a)-\theta(u ; q, a)-\frac{u \delta}{\phi(q)}\right\}^{2} d u=\int_{p_{n}}^{p_{n+1}-\frac{B}{2}}\left(\frac{u \delta}{\phi(q)}\right)^{2} d u \gg \frac{\delta^{2} x^{2}}{\phi(q)^{2}} d_{n}
$$

Hence $d_{n} \ll \sqrt{\epsilon \phi(q) x \log x}$. According to Theorem 2 with the above choice of $\delta$ this holds uniformly for $q \leq x^{\frac{1}{3}-\eta}$ for any $\eta>0$ fixed. To conclude the proof of Corollary 3 we let $\epsilon \rightarrow 0^{+}$sufficiently slowly. 
To prove Corollary 4 it is suitable to have a version of Theorem 2 with fixed difference $H$ rather than the varying difference $u \delta$ in the integrand. Applying the method of Goldston and Montgomery [4] one obtains, for $H \rightarrow \infty$ with $x$ and $q \leq(\log x)^{A}$,

$$
\int_{x}^{2 x}\left\{\theta(u+H ; q, a)-\theta(u ; q, a)-\frac{H}{\phi(q)}\right\}^{2} d u=(1+o(1)) \frac{H x}{\phi(q)} \log \frac{q x}{H} .
$$

Remark: In deriving (15) one uses the unconditional estimate

$$
\int_{x}^{2 x}\left\{\theta(u+u \delta ; q, a)-\theta(u ; q, a)-\frac{u \delta}{\phi(q)}\right\}^{2} d u \ll \frac{x^{3} \delta^{2}}{\phi(q)^{2}}+\frac{\delta x^{2}}{\phi(q)} \log x,
$$

which is valid if $0 \leq \delta \leq 1$ and $q<x^{1-\varepsilon}$, for the integration over $0 \leq \delta \leq \frac{1}{x}$.

Expanding the integrand in (15) and using the Siegel-Walfisz theorem yields

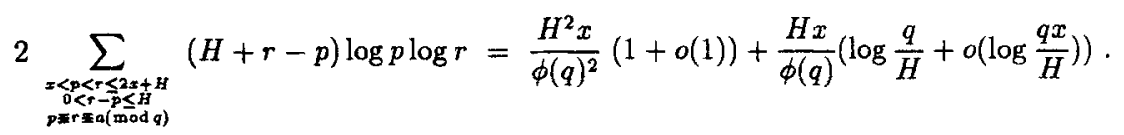

With the choice $H=\epsilon \phi(q) \log x$ the right-hand side is a positive quantity for any fixed $\epsilon>0$ if $x$ is sufficiently large. This completes the proof of Corollary 4.

\section{Acknowledgement}

I thank Professor J.B. Friedlander, my thesis advisor, for his encouragement and comments while I worked on this topic at the University of Toronto. I am grateful to Professor D.A. Goldston for the argument used in the proof of Corollary 3.

\section{References}

[1] H. Davenport, Multiplicative Number Theory (2nd ed.) Springer-Verlag, New York, 1980

[2] D.A. Goldston, Large differences between consecutive prime numbers, Thesis, University of California, Berkeley, 1981

[3] D.A. Goldston and D.R. Heath-Brown, A note on the differences between consecutive primes, Math. Ann., 260 (1984), 317-320

[4] D.A. Goldston and H.L. Montgomery, Pair correlation and primes in short intervals, Analytic Number Theory and Diophantine Problems, Proceedings of a Conference at Oklahoma State University, Birkhauser, Boston, 1987, 183-203

[5] D.R. Heath-Brown, Gaps between primes, and the pair correlation of zeros of the zetafunction, Acta Arith. 41 (1982), 85-99

[6] E. Landau, Handbuch der Lehre von der Verteilung der Primzahlen, Teubner, Berlin, 1909.

[7] H.L. Montgomery, The pair correlation of zeros of the zeta function, Analytic Number Theory, Proc. Sympos. Pure Math. 24 (1973), 181-193

[8] H.L. Montgomery and R.C. Vaughan, Hilbert's inequality, J. London Math. Soc. (2) 8 (1974), 73-82

Mathematics Department

Bilkent University

Ankara, 06533, Turkey 